\title{
Loss of fragile histidine triad (Fhit) protein expression alters the translation of cancer-associated mRNAs
}

\author{
Daniel L. Kiss ${ }^{1,2}$, William D. Baez ${ }^{1,3}$, Kay Huebner ${ }^{4}$, Ralf Bundschuh ${ }^{1,3,5,6}$ and Daniel R. Schoenberg ${ }^{1,2^{*}}$ (D)
}

\begin{abstract}
Objectives: In $>50 \%$ of cancers tumor development involves the early loss of Fhit (fragile histidine triad) protein expression, yet the mechanistic pathway(s) by which Fhit mediates its tumor suppressor functions are not fully understood. Earlier attempts to identify a Fhit-deficient gene expression profile relied on total cellular RNA and microarray analysis. The data here used RNA sequencing (RNA-Seq) of Fhit-negative and Fhit-positive cells as proof of principle for the impact of Fhit on specific mRNAs, and to lay the foundation for a study using ribosome profiling to identify mRNAs whose translation is affected by FHIT loss.

Data description: RNA-Seq was performed on RNA from lines of Fhit-expressing and Fhit-deficient lung cancer cells. This identified changes in the levels of mRNAs for a number of cell survival and cell cycle progression genes. Polysome profile analysis performed on cytoplasmic extracts from Fhit-negative and Fhit-positive cells showed changes in the sedimentation of select mRNAs consistent with changes in translation efficiency. The impact of differential Fhit expression on the turnover of selected cancer-linked mRNAs was determined by RT-qPCR of cytoplasmic RNA isolated at intervals after treating cells with a transcription inhibitor.
\end{abstract}

Keywords: Fhit, Translational control, Cancer, mRNA sequencing, Polysome gradients, Tumor suppressor

\section{Objective}

The expression of the fragile histidine triad gene, FHIT, is lost in over half of human cancers [1]. Because Fhit is a cytoplasmic protein with few interacting protein partners, finding both the mechanism and downstream effectors of Fhit genome protective and tumor suppressor activities have been challenging $[1,2]$. Early experiments to characterize the enzymatic function(s) of Fhit showed that Fhit binds and cleaves diadenosine triphosphate (ApppA) and other dinucleoside triphosphates in vitro $[3,4]$. The ApppA molecules are very similar to free cap dinucleotides $(\mathrm{m} 7 \mathrm{GpppN})$. All mRNAs receive a $5^{\prime}$ cap consisting of 7-methylguanosine linked to the first transcribed nucleotide through a $5^{\prime}, 5^{\prime}$ triphosphate linkage. Cap dinucleotides are generated as end-products of the

\footnotetext{
*Correspondence: schoenberg.3@osu.edu

${ }^{1}$ Center for RNA Biology, The Ohio State University, 484 West 12th Ave., Columbus, $\mathrm{OH}$ 43210, USA

Full list of author information is available at the end of the article
}

$3^{\prime}-5^{\prime}$ RNA decay pathway. In normal cells free cap dinucleotides are degraded by scavenger decapping enzymes [5]. Interestingly, Fhit has been shown to bind and hydrolyze cap dinucleotides in vitro [6]. The subsequent discovery that Fhit and its yeast homolog Aph1 exhibit scavenger decapping activity, at least in vitro, suggests a mechanism through which it may act as a tumor suppressor and genome caretaker, and may be key to identifying additional downstream effectors [7]. We tested the hypothesis that Fhit regulates cancer linked mRNAs at the translational level. Our initial work showing that TK1 protein expression is translationally controlled by Fhit is consistent with a scavenger decapping-based regulatory mechanism [8]. The current study used RNA-Seq, polysome profiling and mRNA turnover analysis to identify Fhit-mediated changes in additional cancer-linked mRNAs. This laid the foundation for a subsequent study to identify the transcriptome-wide impact of FHIT loss on translation [9]. In the latter study ribosome profiling 
Table 1 Overview of data files/data sets

\begin{tabular}{|c|c|c|c|}
\hline Label & Name of data file/data set & File types (file extension) & $\begin{array}{l}\text { Data repository and identifier (DOI or accession } \\
\text { number) }\end{array}$ \\
\hline Data set 1 & RNA-Seq FASTQ files generated from RNA sequencing & FASTQ files & $\begin{array}{l}\text { [10] https://www.ncbi.nlm.nih.gov/Traces/study } \\
\text { /?acc=SRP124332 }\end{array}$ \\
\hline Data file 2 & $\begin{array}{l}\text { Changes in mRNA levels upon Fhit rescue as assayed } \\
\text { by RNA-Seq }\end{array}$ & MS Excel file (.xIsx) & [11] http://dx.doi.org/10.6084/m9.figshare.5539123 \\
\hline Data file 3 & $\begin{array}{l}\text { Polysome gradient analysis of translational changes in } \\
\text { response to Fhit loss }\end{array}$ & Adobe acrobat file (.pdf) & [12] http://dx.doi.org/10.6084/m9.figshare.5540473 \\
\hline Data file 4 & Numerical qPCR values and calculations for Data file 3 & MS Excel file (.xIsx) & [13] http://dx.doi.org/10.6084/m9.figshare.5877033 \\
\hline Data file 5 & $\begin{array}{l}\text { Quantifying changes in ribosome occupancy when } \\
\text { Fhit expression is lost }\end{array}$ & Adobe acrobat file (.pdf) & [14] http://dx.doi.org/10.6084/m9.figshare.5540146 \\
\hline Data file 6 & $\begin{array}{l}\text { Assaying changes in mRNA stability in response to } \\
\text { Fhit loss }\end{array}$ & Adobe acrobat file (.pdf) & [15] http://dx.doi.org/10.6084/m9.figshare.5540476 \\
\hline Data file 7 & Numerical qPCR values and calculations for Data file 6 & MS Excel file (.x|sx) & [16] http://dx.doi.org/10.6084/m9.figshare.5877027 \\
\hline Data file 8 & $\begin{array}{l}\text { The methods used for the experiments and data } \\
\text { analysis }\end{array}$ & Adobe acrobat file (.pdf) & [17] http://dx.doi.org/10.6084/m9.figshare.5540482 \\
\hline
\end{tabular}

and an independent set of sample-matched RNA-Seq data were used to identify Fhit-mediated changes in ribosome occupancy.

\section{Data description}

The data in this submission come from three different experiments. The first experiment (Data files 1 and 2) is an RNA-Seq gene expression study to characterize global Fhit-mediated changes in gene expression in cytoplasmic RNA harvested from H1299 lung cancer cells where Fhit is expressed or silenced. With a padj significance threshold of $<0.05$, the RNA-Seq libraries yielded $\sim 900$ mRNAs with levels of detection differing in cytoplasm by at least 1.5-fold (Data file 2) in Fhit positive vs. Fhit negative H1299 cells.

Next, we assessed the translation of several newly identified Fhit-affected mRNAs using cDNAs remaining from earlier cytoplasmic polysome gradient fractionation experiments [8]. Polysome gradient fractionation separates protein-RNA complexes based on their mass, and can approximate the translational output of an mRNA based on the number of ribosomes loaded onto it. A total of ten mRNAs, two controls (Rplp0 and PolR2A), and eight cancer-linked mRNAs (ATF6, GDF15, CENPH, CSK1B, SPC25, AURKA, NDC80, and Top2A) were selected from our RNA-Seq data and assessed for changes in translation. The resulting data are presented in Data files 3, 4, and 5 (Table 1).

Finally, we determined if Fhit expression altered the stability of the mRNAs studied. Briefly, cells were treated with 5,6-dichloro-1- $\beta$-D-ribofuranosylbenzimidazole (DRB), a selective inhibitor of RNA polymerase II, for 0-10 h. RNA was harvested from cytoplasmic extracts at $2 \mathrm{~h}$ intervals after DRB treatment began. Fhit-dependent changes in the stability of targeted mRNAs was assayed using RT-qPCR and are reported in Data files 6 and 7.

A detailed description of the methods used to gather and analyze these data is provided in Data file 6.

\section{Limitations}

The key limitation of this study is the small cross section of mRNAs assayed. Of the $\sim 900$ mRNAs that changed $\geq 1.5$-fold in response to Fhit loss, we assessed 10 , two controls and eight mRNAs whose gene products were known to be involved in cancer-linked processes. Since our previous work showed that Fhit affects the translation of TK1 mRNA [8] we assayed these 10 mRNAs for effects on translation and mRNA stability. Ideally, we would have assayed more targets. The second limitation was the behavior of our two control mRNAs in the polysome gradient experiments. Namely, the translation rates of our control mRNAs (RPLP0 and POLR2A) change when Fhit is lost. Although the behavior of an ideal control would not change with respect to Fhit levels, this result is actually predicted by our hypothesis of how Fhit mediates its translational control functions as a function of its scavenger decapping activity.

\section{Abbreviations}

FHIT: fragile histidine triad gene; Fhit: the FHIT gene protein product; CHX: cycloheximide; DRB: 5,6-dichloro-1- $\beta$-D-ribofuranosylbenzimidazole; RT-qPCR: quantitative reverse transcription polymerase chain reaction; mRNP: messenger ribonucleoprotein.

\section{Authors' contributions}

DLK conceived and designed the study and performed both the cell culture and molecular biology work, including the polysome gradients, isolating RNA for RNA-Seq and RT-qPCR and Western blotting. He also generated all the figures and much of the text of the manuscript. WDB performed the bioinformatics analysis and generated the results used in the table of mRNAs changed by RNA-Seq. Also generated text of the shared methods, and edited and 
approved the manuscript. RB supervised WB's approach to data analysis, participated in aspects of informatics analysis, participated in weekly strategy and planning meetings, and edited and approved the manuscript. KH provided information and insight into the relationship of Fhit to cancer, provided cell lines and antibodies used in this study, and generated text and approved the manuscript. DRS oversaw planning and experimental approach, supervised DLK's experimental design and analysis, participated in weekly strategy and planning meetings, and edited and approved the manuscript. All authors read and approved the final manuscript.

\begin{abstract}
Author details
${ }^{1}$ Center for RNA Biology, The Ohio State University, 484 West 12th Ave., Columbus, OH 43210, USA. ${ }^{2}$ Department of Biological Chemistry and Pharmacology, The Ohio State University, 1060 Carmack Rd., Columbus, OH 43210 USA. ${ }^{3}$ Department of Physics, The Ohio State University, 191 West Woodruff Ave., Columbus, OH 43210, USA. ${ }^{4}$ Department of Cancer Biology and Genetics, The Ohio State University, 460 West 12 Ave., Columbus, OH 43210, USA. ${ }^{5}$ Department of Chemistry and Biochemistry, The Ohio State University, Columbus, OH 43210, USA. ${ }^{6}$ Division of Hematology, Department of Internal Medicine, The Ohio State University, Columbus, OH 43210, USA.
\end{abstract}

\section{Acknowledgements}

The content is solely the responsibility of the authors and does not necessarily represent the official views of Pelotonia, The Ohio State University, the National Institutes of Health or the National Science Foundation.

\section{Competing interests}

The authors declare that they have no competing interests.

\section{Availability of data materials}

The data described in this Data note can be freely and openly accessed on [NCBI Sequence Read Archive (SRA) Data set 1: https://www.ncbi.nlm.nih. gov/Traces/study/?acc=SRP124332, and on Figshare: Data file 2: http://dx.doi. org/10.6084/m9.figshare.5539123. Data file 3: http://dx.doi.org/10.6084/ m9.figshare.5540473. Data file 4: http://dx.doi.org/10.6084/m9.figshare.58770 33. Data file 5: http://dx.doi.org/10.6084/m9.figshare.5540146. Data file 6 : http://dx.doi.org/10.6084/m9.figshare.5540476. Data file 7: http://dx.doi. org/10.6084/m9.figshare.5877027. Data file 8: http://dx.doi.org/10.6084/ m9.figshare.5540482].

\section{Consent for publication}

Not applicable.

\section{Ethics approval and consent to participate}

Not applicable.

\section{Funding}

This research was supported by: a Pelotonia postdoctoral fellowship (to DLK) and a Pelotonia multi-PI IDEA grant from the OSUCCC Pelotonia fund (to $\mathrm{KH}$, DRS, RB), and grants from the National Institute of General Medical Science (GM084177 to DRS), from the National Cancer Institute (CA120516 and CA154200 to KH), and the National Science Foundation (DMR-1410172 and DMR-1719316 to RB).

\section{Publisher's Note}

Springer Nature remains neutral with regard to jurisdictional claims in published maps and institutional affiliations.
Received: 7 November 2017 Accepted: 7 March 2018

Published online: 14 March 2018

\section{References}

1. Karras JR, Schrock MS, Batar B, Huebner K. Fragile genes that are frequently altered in cancer: players not passengers. Cytogenet Genome Res. 2016:150(3-4):208-16.

2. Saldivar JC, Miuma S, Bene J, Hosseini SA, Shibata H, Sun J, Wheeler LJ, Mathews CK, Huebner K. Initiation of genome instability and preneoplastic processes through loss of Fhit expression. PLoS Genet. 2012;8(11):e1003077.

3. Brenner C, Bieganowski P, Pace HC, Huebner K. The histidine triad superfamily of nucleotide-binding proteins. J Cell Physiol. 1999;181(2):179.

4. Brenner C. Hint, Fhit, and GalT: function, structure, evolution, and mechanism of three branches of the histidine triad superfamily of nucleotide hydrolases and transferases. Biochemistry. 2002;41(29):9003-14.

5. Liu H, Rodgers ND, Jiao X, Kiledjian M. The scavenger mRNA decapping enzyme DcpS is a member of the HIT family of pyrophosphatases. EMBO J. 2002;21(17):4699-708.

6. Draganescu A, Hodawadekar SC, Gee KR, Brenner C. Fhit-nucleotide specificity probed with novel fluorescent and fluorogenic substrates. J Biol Chem. 2000;275(7):4555-60.

7. Taverniti $V$, Séraphin B. Elimination of cap structures generated by mRNA decay involves the new scavenger mRNA decapping enzyme Aph1/FHIT together with DcpS. Nucleic Acids Res. 2015;43(1):482-92.

8. Kiss DL, Waters CE, Ouda IM, Saldivar JC, Karras JR, Amin ZA, Mahrous S, Druck T, Bundschuh RA, Schoenberg DR, Huebner K. Identification of Fhit as a post-transcriptional effector of thymidine kinase 1 expression. Biochim Biophys Acta. 2017;1860(3):374-82

9. Kiss DL, Baez W, Huebner K, Bundschuh R, Schoenberg DR. Impact of FHIT loss on the translation of cancer-associated mRNAs. Mol Cancer. 2017;16(1):179

10. NCBI Sequence Read Archive SRP124332. 2018.

11. Kiss DL, Baez W, Huebner K, Bundschuh R, Schoenberg DR. Figshare. 2018. http://dx.doi.org/10.6084/m9.figshare.5539123.

12. Kiss DL, Baez W, Huebner K, Bundschuh R, Schoenberg DR. Figshare. 2018 http://dx.doi.org/10.6084/m9.figshare.5540473.

13. Kiss DL, Baez W, Huebner K, Bundschuh R, Schoenberg DR. Figshare. 2018 http://dx.doi.org/10.6084/m9.figshare.5540146.

14. Kiss DL, Baez W, Huebner K, Bundschuh R, Schoenberg DR. Figshare. 2018. http://dx.doi.org/10.6084/m9.figshare.5540146.

15. Kiss DL, Baez W, Huebner K, Bundschuh R, Schoenberg DR. Figshare. 2018 http://dx.doi.org/10.6084/m9.figshare.5540476.

16. Kiss DL, Baez W, Huebner K, Bundschuh R, Schoenberg DR. Figshare. 2018 http://dx.doi.org/10.6084/m9.figshare.5877027.

17. Kiss DL, Baez W, Huebner K, Bundschuh R, Schoenberg DR. Figshare. 2018 http://dx.doi.org/10.6084/m9.figshare.5540482.

\section{Submit your next manuscript to BioMed Central and we will help you at every step:}

- We accept pre-submission inquiries

- Our selector tool helps you to find the most relevant journal

- We provide round the clock customer support

- Convenient online submission

- Thorough peer review

- Inclusion in PubMed and all major indexing services

- Maximum visibility for your research

Submit your manuscript at www.biomedcentral com/submit
Ciomed Central 